\title{
Depressão pós-parto e alterações de sono aos 12 meses em bebês nascidos na zona urbana da cidade de Pelotas/RS
}

Postpartum depression and sleep disorders in 12 month-old babies born in the urban area of Pelotas city

Eliane Rozales Lopes', Karen Jansen¹, Luciana de Ávila Quevedo', Russélia Godoy Vanila', Ricardo Azevedo da Silva', Ricardo Tavares Pinheiro'

\section{RESUMO}

Objetivo: Verificar se existe associação entre as alterações no sono dos bebês aos 12 meses de vida e a depressão pós-parto materna. Métodos: Estudo do tipo transversal aninhado a uma coorte. A amostra foi constituída por mulheres que realizaram o acompanhamento pelo Sistema Único de Saúde (SUS), nas unidades básicas de saúde do município de Pelotas, e que tiveram seus partos a partir de junho/2006. Os bebês de 12 meses oriundos dessa gestação também fazem parte da amostra. Para avaliar a presença de sintomas depressivos nas mães, foi utilizada a Edinburgh Postnatal Depression Scale (EPDS) e foram investigados os seguintes comportamentos do sono dos bebês: horas de sono por dia, regularidade do horário para dormir e acordar, sono agitado e despertar noturno. Para análise, foi utilizada Regressão de Poisson. Resultados: $35,7 \%$ dos bebês possuem alteração no padrão de sono. Após o ajuste ao mode-

\section{Palavras-chave}

Depressão puerperal, distúrbios do sono, criança, interação mãe-criança. lo hierárquico proposto, a alteração no sono infantil manteve associação com a sintomatologia depressiva da mãe $(p \leq 0,01)$. Conclusões: Os profissionais de saúde devem investigar rotineiramente os comportamentos de sono dos bebês e dar atenção à saúde mental das mães, a fim de identificar os problemas precocemente e oferecer suporte no manejo do sono dos bebês.
1 Universidade Católica de Pelotas (UCPel), Programa de Pós-Graduação em Saúde e Comportamento.

\begin{abstract}
Objective: Verify whether there is association between sleep disorders in babies at 12 months of age and postpartum depression in motherhood. Methods: Cross sectional study. The sample was made up of women who had done their prenatal medical care at the National Health System (SUS), at the health basic units in Pelotas and who had their deliveries from June, 2006. The 12 month old babies from these women are also part of the sample. In order to assess depressive symptoms in the mothers, Edinburgh Postnatal Depression Scale was used and the following sleeping behaviors of the babies were investigated: hours of sleep per day, regularity of sleep and wake up time, disturbed sleep and night awakening. Poisson Regression was used for the analysis. Results: $35.7 \%$ of the babies showed alterations in their sleeping patterns. After adjusting for the proposed
\end{abstract}

Endereço para correspondência: Eliane Rozales Lopes Rua Emilio Jorge dos Reis, 512, Três Vendas - 96020-440 - Pelotas, RS Telefones: 55 (53) 3303-0474/55 (53) 9112-5240

E-mail: elirlopes@yahoo.com.br 


\section{Keywords}

Perinatal depression, sleep disorders, child, interaction mother-child. hierarchal model, sleep alteration of the babies was still associated with the depressive symptoms of the mothers $(p \leq 0,01)$. Conclusions: Health professionals should regularly investigate sleeping behaviors of babies and pay attention to mothers' mental health in order to identify problems early and offer support in the management of babies' sleep.

\section{INTRODUÇÃO}

O sono é uma função biológica que serve para restaurar o sistema imunitário, as capacidades mentais e recuperar as energias gastas durante a vigília'.

As alterações do sono em crianças são a quinta causa de relatos de preocupações dos pais em consultórios pediátri$\cos ^{2}$ e, por esse motivo, o assunto vem ganhando relevância no meio científico.

Embora os bebês possam apresentar variações individuais quanto ao padrão de sono, aos 12 meses eles dormem em média 12 horas por dia e, em geral, têm a regularidade e a previsibilidade do sono. Já apresentam os padrões de sono noturno definidos e também podem fazer a sesta com horários previsíveis. A sesta dura aproximadamente duas horas e vai começar a desaparecer aos 2 anos de vida ${ }^{3-7}$.

Existe divergência quanto à prevalência dos distúrbios de sono em bebês, variando de $14 \%{ }^{8}$ a $46 \%{ }^{9}$. Isso ocorre, provavelmente, por não existir um consenso na literatura sobre o que pode ser objetivamente considerado uma alteração de sono infantil nesse período.

Diferentes fatores atuam sobre o sono dos bebês, entre eles estão as causas constitucionais (genéticas), o contexto familiar, as condições sociais e a vida psíquica do próprio bebê. Na criança até 2 anos de idade, o sono, frequentemente, será porta-voz da qualidade da relação entre a criança e seus pais. A mãe é proposta como a "guardiã" do sono do seu bebê ${ }^{4}$, entretanto nem sempre as mães conseguem exercer esse papel, principalmente quando na presença de sintomatologia depressiva. A mãe deprimida apresentará dificuldades de perceber e interpretar as necessidades da criança ${ }^{10}$.

A depressão é um transtorno incapacitante que pode atingir, em nosso meio, até 25\% das mães e $11 \%$ dos pais no primeiro ano de vida da criança ${ }^{11}$.

Existe a indicação de que os bebês de mães deprimidas podem apresentar alteração dos padrões de sono ${ }^{10}$. Estudos referem que existe associação entre os sintomas depressivos maternos e a frequência dos despertares ${ }^{6,9,12-15}$, a qualidade do sono e a quantidade de tempo durante o qual a criança dorme ${ }^{12,15}$.

Os estudos brasileiros que associam problemas de sono em bebês à depressão materna ainda são insipientes, e os resultados encontrados por investigações de outros países não devem ser generalizados para o Brasil, em virtude das diferenças culturais na atenção, cuidado e manejo dos bebês.
As crianças com problemas de sono mostram-se mais cansadas, desatentas, irritadiças e têm maiores dificuldades para modular seus impulsos e emoções ${ }^{9}$. Essa associação entre problemas de sono e alterações comportamentais na infância têm sido relacionada a problemas de desenvolvimento em distintas áreas (cognitiva, linguagem, comportamental) aos 2 e 3 anos de idade e na adolescência ${ }^{16,17}$.

Torna-se relevante identificar precocemente as alterações de sono e os fatores associados, a fim de minimizar os efeitos negativos sobre o desenvolvimento da criança, bem como proporcionar o bem-estar familiar.

O objetivo desta investigação é verificar se existe associação entre as alterações no sono dos bebês aos 12 meses de vida e a depressão materna.

\section{MÉTODOS}

Este é um estudo do tipo transversal aninhado a uma coorte. A amostra foi constituída por mulheres que realizaram o acompanhamento pelo Sistema Único de Saúde (SUS), nas unidades básicas de saúde do município de Pelotas, e tiveram seus partos a partir de junho/2006. Os bebês de 12 meses oriundos dessas gestações também fazem parte da amostra.

Para o cálculo amostral, utilizou-se um poder de $80 \%$ e o nível de confiança de 95\%. Foram consideradas a menor prevalência de depressão encontrada na literatura (13\%) e a prevalência de alterações do sono de bebês de mães não deprimidas $(36 \%)$ e OR $(2,88)$ encontradas por Hiscock e Wake 9 . Chegou-se ao N de 277 díades (mães e filhos aos 12 meses), entretanto foi acrescentado 30\% para controlar as perdas, recusas e os fatores de confusão, sendo necessário captar, no mínimo, 360 díades.

Neste estudo transversal, as mães e os pais dos bebês foram visitados em suas residências no mês em que o bebê completou 12 meses de idade. Ambos os pais receberam informações a respeito dos objetivos da pesquisa e assinaram um Consentimento Livre e Esclarecido. Após esse procedimento, eles responderam a um questionário autoaplicado, com variáveis socioeconômicas sobre o parto e o primeiro ano de vida do bebê. As informações a respeito do peso do bebê ao nascer e a idade gestacional foram retiradas da "carteirinha do bebê".

Com base na literatura ${ }^{3-7}$, considerou-se que comportamentos disfuncionais de sono aos 12 meses são: dormir me- 
nos ou mais que 12 horas por dia, incluindo as sestas; acordar uma vez ou mais durante a noite; não possuir horário para dormir e acordar; ter um sono agitado. Os bebês que falavam, gritavam, tinham pesadelos ou mudavam de lugar enquanto dormiam foram definidos como tendo um sono agitado.

Os bebês que despertavam ao menos uma vez durante a noite e que possuíam um sono agitado, concomitantemente, foram considerados como tendo uma alteração de sono, sendo esse o nosso desfecho. Definiram-se essas variáveis, pois elas foram as únicas a possuir associação com a depressão materna.

Para avaliar a presença de sintomas depressivos, foi utilizada a EPDS, escala que contém 10 questões em formato de respostas do tipo Likert. Os entrevistados escolhem as respostas que melhor descrevem o modo como eles têm se sentido na última semana de acordo com a intensidade dos sintomas. Foi utilizado o ponto de corte de 10 pontos para o screening da depressão e, sendo assim, considerou-se depressão pós-parto nos indivíduos que pontuaram 10 ou mais na EPDS, no estudo de validação do instrumento, realizado na cidade de Pelotas, RS. Com esse ponto de corte, a sensibilidade é de $82,6 \%$ e a especificidade, de $65,4 \%{ }^{18}$.

Após a revisão de literatura, os possíveis confundidores foram: idade materna, situação socioeconômica, escolaridade da mãe, morar com companheiro, trabalhar fora de casa atualmente, peso do bebê ao nascer, sexo, prematuridade, problemas ou síndrome no nascimento, número de vezes em que adoeceu no último mês, número de consultas médicas no primeiro ano de vida, ocorrência de algum acidente, aleitamento materno, se dorme no quarto dos pais, permanência ou não na creche e depressão de ambos os pais.

Para avaliar o nível socioeconômico, utilizou-se a classificação da Associação Brasileira de Empresas de Pesquisa (ABEP) ${ }^{19}$, que é baseada na acumulação de bens materiais e na escolaridade do chefe da família, classificando os sujeitos em cinco classes (A, B, C, D e E) de acordo com o escore obtido.

Investigou-se a presença de algum tipo de problema do bebê logo após o nascimento; os problemas relatados pelos pais foram: má-formação congênita, doenças do aparelho respiratório, gastrointestinal, cardiovascular, endócrino ou neurológico.

Após a coleta de dados, foram realizadas duas digitações no programa EPI-INFO 6.04d e estas foram comparadas para que as inconsistências fossem solucionadas. Foram utilizados o pacote estatístico SPSS 10.0 for Windows e o STATA 9 para a análise dos dados. Foi feita análise univariada para conhecer as características das mães e bebês entrevistados. Na análise bivariada, foi utilizado o teste do qui-quadrado para comparar prevalências.

Pelo fato de as alterações de sono infantil serem influenciadas por diversos fatores, foi realizada uma análise multivariada por Regressão de Poisson. A análise multivariada foi realizada seguindo um modelo hierárquico previamente proposto. O modelo foi composto, no primeiro nível, pela idade materna, nível socioeconômico, escolaridade e estado civil; no segundo nível, pelo sexo do bebê, peso ao nascer, prematuridade, problemas no nascimento ou presença de síndromes, número de vezes em que adoeceu no último mês, número de consultas no primeiro ano de vida, acidentes sofridos pelo bebê, aleitamento materno, permanência ou não na creche e trabalho materno atualmente; e no terceiro nível, pela depressão materna e paterna.

O critério para inclusão das variáveis no modelo final foi possuir um $p \leq 0,20$ no teste de razões de verossimilhança; estas permaneceram no modelo e as demais foram excluídas. Nesse tipo de modelo, as variáveis situadas em um nível hierárquico superior ao da variável em questão são consideradas como potenciais confundidoras da relação entre essa variável e o desfecho em estudo, enquanto as variáveis em níveis inferiores são consideradas como potenciais mediadoras da associação.

A investigação foi aprovada pelo comitê local de ética em pesquisa da Universidade Católica de Pelotas, de acordo com as normas vigentes (CONEP - Resolução no 196/96).

\section{RESULTADOS}

A investigação avaliou uma amostra representativa de 409 díades. As recusas ou perdas representaram 8\% (36).

A amostra do presente estudo caracteriza-se por mães de baixa classe social; a maioria encontra-se no nível socioeconômico C, D ou E (85,9\%), tem em média 26 anos (DP $\pm 6,57)$ e é casada ou vive com o companheiro (76,1\%). Quanto à escolaridade, $37,3 \%$ delas têm até o primeiro grau incompleto. Das muIheres entrevistadas, 65,6\% não trabalham fora de casa e 6,4\% deixam os filhos em alguma creche. Dessas mães, 19,5\% apresentaram sintomas depressivos e $11 \%$ dos pais dos seus bebês também possuíam screening para esse quadro (Tabela 1).

Quanto aos bebês, a maior parte é do sexo masculino $(53,3 \%)$, nasceu com mais de 38 semanas de gestação $(54,3 \%)$, sem nenhum problema ou síndrome $(89,7 \%)$ e com o peso acima de $2.499 \mathrm{~g}(94,3 \%)$. Em relação à saúde dessas crianças, os pais relataram que $90,7 \%$ não ficaram doentes nenhuma vez no último mês, $78,5 \%$ levaram os seus filhos até 12 vezes ao médico durante esse primeiro ano de vida e $38,8 \%$ sofreram algum tipo de acidente (quedas, queimaduras ou luxações) desde o nascimento. Trinta e oito crianças $(10,3 \%)$ apresentaram algum tipo de problema logo após o nascimento e entre esses problemas estão as más-formações congênitas, doenças do aparelho respiratório, endócrino, cardiovascular, neurológico, entre outros. Dos bebês da amostra, 37,1\% recebem aleitamento materno com 1 ano de idade (Tabela 1); 33,9\% das crianças dormem na cama dos pais e $57 \%$ dormem no mesmo ambiente que os pais, mas em camas separadas. Essa variável não se associou à depressão materna nem ao relato das alterações de sono $(p \geq 0,05)$. 
Tabela 1. Distribuição da amostra e análise bruta e ajustada ao modelo hierárquico do desfecho alterações de sono de bebês aos 12 meses, por Regressão de Poisson (risco relativo e 95\% de intervalo de confiança)

\begin{tabular}{|c|c|c|c|c|c|}
\hline Variáveis independentes & $\mathrm{N}(\%)$ & $\begin{array}{l}\text { Alteraçöes de sono } \\
\text { RP bruta (IC } 95 \%)\end{array}$ & p-valor* & $\begin{array}{l}\text { Alteraçōes do sono } \\
\text { RP ajustada (IC 95\%) }\end{array}$ & $\mathrm{p}$-valor** \\
\hline $1^{\circ}$ Nível & & & & & \\
\hline $\begin{array}{l}\text { Idade materna (anos) } \\
\text { Até } 19 \text { anos } \\
20 \text { a } 35 \text { anos } \\
36 \text { anos ou mais }\end{array}$ & $\begin{array}{l}63(15,8 \%) \\
293(73,3 \%) \\
44(11,0 \%)\end{array}$ & $\begin{array}{l}1,22(0,76 ; 1,94) \\
0,84(0,55 ; 1,28) \\
1,00\end{array}$ & 0,247 & - & - \\
\hline $\begin{array}{l}\text { Nível socioeconômico } \\
\text { A e B } \\
\text { C } \\
\text { DeE }\end{array}$ & $\begin{array}{l}49(14,1 \%) \\
175(50,4 \%) \\
123(35,5 \%)\end{array}$ & $\begin{array}{l}1,00 \\
1,11(0,68 ; 1,81) \\
1,13(0,68 ; 1,88)\end{array}$ & 0,666 & - & - \\
\hline $\begin{array}{l}\text { Anos de escolaridade da mãe } \\
0 \text { a } 7 \text { anos } \\
8 \text { a } 11 \text { anos } \\
12 \text { a } 15 \text { anos } \\
16 \text { anos ou mais }\end{array}$ & $\begin{array}{l}145(37,3 \%) \\
89(23,1 \%) \\
137(35,6 \%) \\
14(3,6 \%)\end{array}$ & $\begin{array}{l}1,00 \\
1,38(0,99 ; 1,93) \\
0,92(0,65 ; 1,31) \\
1,06(0,50 ; 2,23)\end{array}$ & 0,704 & - & - \\
\hline $\begin{array}{l}\text { Mãe vive com companheiro } \\
\text { Sim } \\
\text { Não }\end{array}$ & $\begin{array}{l}302(76,1 \%) \\
95(23,9 \%)\end{array}$ & $\begin{array}{l}1,00 \\
1,19(0,89 ; 1,60)\end{array}$ & 0,240 & - & - \\
\hline $2^{\circ}$ Nível & & & & & \\
\hline $\begin{array}{l}\text { Sexo } \\
\text { Masculino } \\
\text { Feminino }\end{array}$ & $\begin{array}{l}216(53,3 \%) \\
189(46,7 \%)\end{array}$ & $\begin{array}{l}1,02(0,77 ; 1,34) \\
1,00\end{array}$ & 0,894 & - & - \\
\hline $\begin{array}{l}\text { Peso ao nascer } \\
\text { Menor que } 2.499 \mathrm{~g} \\
\text { Maior que } 2.500 \mathrm{~g}\end{array}$ & $\begin{array}{l}23(5,7 \%) \\
380(94,3 \%)\end{array}$ & $\begin{array}{l}1,00 \\
1,12(0,60 ; 1,11)\end{array}$ & 0,715 & - & - \\
\hline $\begin{array}{l}\text { Prematuridade } \\
\text { Sim } \\
\text { Não }\end{array}$ & $\begin{array}{l}176(45,7 \%) \\
209(54,3 \%)\end{array}$ & $\begin{array}{l}1,19(0,90 ; 1,56) \\
1,00\end{array}$ & 0,214 & - & - \\
\hline $\begin{array}{l}\text { Problemas no nascimento } \\
\text { Sim } \\
\text { Não }\end{array}$ & $\begin{array}{l}41(10,3 \%) \\
356(89,7 \%)\end{array}$ & $\begin{array}{l}1,30(0,88 ; 1,90) \\
1,00\end{array}$ & 0,181 & $\begin{array}{l}1,30(0,89 ; 1,90) \\
1,00\end{array}$ & 0,177 \\
\hline $\begin{array}{l}\text { № de vezes que adoeceu no último mês } \\
\text { Nenhuma ou } 1 \text { vez } \\
2 \text { vezes } \\
3 \text { ou mais vezes }\end{array}$ & $\begin{array}{l}362(90,7 \%) \\
28(7,0 \%) \\
9(2,3 \%)\end{array}$ & $\begin{array}{l}1,00 \\
1,55(1,05 ; 2,30) \\
1,66(0,91 ; 3,04)\end{array}$ & 0,009 & $\begin{array}{l}1,00 \\
1,53(1,01 ; 2,33) \\
1,30(0,67 ; 1,99)\end{array}$ & 0,066 \\
\hline $\begin{array}{l}\text { № de consultas no } 1^{\circ} \text { ano } \\
\text { Até } 12 \\
13 \text { ou mais }\end{array}$ & $\begin{array}{l}303(78,5 \%) \\
83(21,5 \%)\end{array}$ & $\begin{array}{l}1,00 \\
1,05(0,76 ; 1,45)\end{array}$ & 0,772 & - & - \\
\hline $\begin{array}{l}\text { Sofreu algum tipo de acidente } \\
\text { Sim } \\
\text { Não }\end{array}$ & $\begin{array}{l}157(38,8 \%) \\
248(61,2 \%)\end{array}$ & $\begin{array}{l}1,52(1,12 ; 2,06) \\
1,00\end{array}$ & 0,007 & $\begin{array}{l}1,46(1,07 ; 1,99) \\
1,00\end{array}$ & 0,015 \\
\hline $\begin{array}{l}\text { Amamentação atualmente } \\
\text { Sim } \\
\text { Não }\end{array}$ & $\begin{array}{l}151(37,1 \%) \\
256(62,9 \%)\end{array}$ & $\begin{array}{l}1,00 \\
1,16(0,87 ; 1,55)\end{array}$ & 0,307 & - & - \\
\hline $\begin{array}{l}\text { Bebê fica na creche } \\
\text { Sim } \\
\text { Não }\end{array}$ & $\begin{array}{l}26(6,4 \%) \\
378(93,6 \%)\end{array}$ & $\begin{array}{l}1,00 \\
1,02(0,57 ; 1,82)\end{array}$ & 0,939 & - & - \\
\hline $\begin{array}{l}\text { Mãe trabalha atualmente } \\
\text { Sim } \\
\text { Não }\end{array}$ & $\begin{array}{l}139(34,4 \%) \\
265(65,6 \%)\end{array}$ & $\begin{array}{l}1,00 \\
1,16(0,86 ; 1,56)\end{array}$ & 0,330 & - & - \\
\hline \multicolumn{6}{|l|}{$3^{\circ}$ Nível } \\
\hline $\begin{array}{l}\text { Depressão paterna } \\
\text { Não } \\
\text { Sim }\end{array}$ & $\begin{array}{l}291(89 \%) \\
36(11 \%)\end{array}$ & $\begin{array}{l}1,00 \\
0,83(0,48 ; 1,44)\end{array}$ & 0,505 & - & - \\
\hline $\begin{array}{l}\text { Depressão materna } \\
\text { Não } \\
\text { Sim }\end{array}$ & $\begin{array}{l}321(80,5 \%) \\
78(19,5 \%)\end{array}$ & $\begin{array}{l}1,00 \\
1,60(1,22 ; 2,10)\end{array}$ & 0,001 & $\begin{array}{l}1,00 \\
1,52(1,15 ; 2,00)\end{array}$ & 0,003 \\
\hline TOTAL & 409 (100\%) & & & & \\
\hline
\end{tabular}

* Apenas as variáveis independentes que possuem $p$-valor $\leq 0,20$ na análise bruta foram incluídas no modelo hierárquico final.

**As variáveis com $p$-valor $\leq 0,05$ possuem associação estatisticamente significativa. 
No que se refere aos comportamentos de sono infantil, $85,6 \%$ dormiam menos ou mais que 12 horas, 39,8\% ainda não possuíam horário para dormir e acordar, 40,2\% possuíam um sono agitado e despertavam ao menos uma vez durante a noite, chegando a acordar cinco vezes na mesma noite (Tabela 2). Os bebês dormem em média 9 horas e 50 minutos (DP $\pm 2,95)$.

Para verificar a associação entre os comportamentos de sono das crianças e o quadro depressivo das mães, foi realizada uma análise bivariada. O despertar noturno e o sono agitado apresentaram associação $(p \leq 0,05)$ com os sintomas depressivos maternos, enquanto não possuir horário para dormir e acordar e o número de horas em que o bebê dorme por dia não estavam associados ( $p \geq 0,05)$ (Tabela 2 ).

Tabela 2. Análise bivariada das alterações de sono de bebês aos 12 meses em relação ao quadro depressivo de suas mães

\begin{tabular}{|c|c|c|c|c|}
\hline \multirow[b]{2}{*}{ Alteraçôes no sono } & \multirow[b]{2}{*}{$\mathrm{N}(\%)$} & \multicolumn{2}{|c|}{ EPDS } & \multirow[b]{2}{*}{ p-valor* } \\
\hline & & $\begin{array}{c}\text { Sim } \\
\mathrm{N}(\%)\end{array}$ & $\begin{array}{l}\text { Não } \\
\mathrm{N}(\%)\end{array}$ & \\
\hline $\begin{array}{l}\text { Não possuir horário } \\
\text { para dormir e } \\
\text { acordar }\end{array}$ & $162(39,8 \%)$ & $41(42,3 \%)$ & $118(39,3 \%)$ & 0,608 \\
\hline Despertar noturno & $162(40,2 \%)$ & $52(53,6 \%)$ & $105(35,5 \%)$ & 0,002 \\
\hline $\begin{array}{l}\text { Horas de sono con- } \\
\text { sideradas menor ou } \\
\text { maior que o ideal }\end{array}$ & $328(85,6 \%)$ & $78(87,2 \%)$ & $245(83,9 \%)$ & 0,485 \\
\hline Sono agitado & $162(40,2 \%)$ & $50(51,5 \%)$ & $107(36,1 \%)$ & 0,007 \\
\hline
\end{tabular}

* As variáveis com $p$-valor $\leq 0,05$ possuem associaçăo estatisticamente significativa.

Um terço (35,7\%) dos bebês com 12 meses possuem algum tipo de alteração no padrão de sono. Na análise bruta, o desfecho associou-se com o número de vezes em que o bebê ficou doente no último mês $(p \leq 0,05)$, se ele sofreu algum tipo de acidente $(p \leq 0,05)$ e a depressão materna $(p \leq 0,001)$ (Tabela 1).

Para a análise ajustada, o modelo hierárquico final foi composto no primeiro nível pela presença de algum problema ou síndrome no nascimento, pelo número de vezes em que o bebê ficou doente no último mês e se ele já sofreu algum tipo de acidente (quedas, luxações, queimaduras) nesse primeiro ano de vida; e no segundo nível, permaneceu apenas a depressão materna.

Após o ajuste por meio do modelo hierárquico proposto, a alteração no sono infantil manteve associações significativas e independentes $(p \leq 0,05)$ com o fato de o bebê já ter sofrido algum acidente e a sintomatologia depressiva da mãe. O número de vezes em que a criança adoeceu no último mês perdeu significância estatística com o desfecho $(p \geq$ 0,05) (Tabela 1).

Os bebês que sofreram queimaduras, quedas ou luxações apresentaram 46\% maior probabilidade de possuírem alterações no sono aos 12 meses, enquanto os bebês de mães com sintomas depressivos apresentaram 52\% maior probabilidade (Tabela 1).

\section{DISCUSSÃO}

Um terço das crianças com 1 ano de idade apresentou dificuldades com o sono, demonstrando ser um problema frequente nessa faixa etária. A prevalência de alterações no sono dos bebês encontrada neste estudo corresponde às

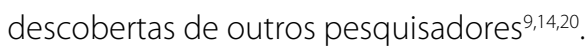

Investigação realizada na cidade de Pelotas avaliou a prevalência do coleito (45,8\%), dos despertares noturnos $(46,1 \%)$ e a média de horas de sono por dia (10 horas) de bebês aos 12 meses e encontrou dados semelhantes ao deste estudo ${ }^{20}$. Porém, a referida investigação não avaliou a depressão materna como fator associado.

As alterações do sono afetam bebês de todas as classes socioeconômicas, independentemente da idade, escolaridade, estado civil e trabalho maternos. As características constitucionais do bebê (sexo, presença de alguma síndrome), problemas perinatais (prematuridade, baixo peso ao nascer) e aspectos da saúde física (número de consultas no primeiro ano de vida, número de vezes em que ficou doente) não estão associados com as alterações de sono aos 12 meses. Há divergências na literatura quanto a esses achados; Bayer et al. ${ }^{14}$ encontraram associação entre problemas de sono de bebês de 3 a 6 meses com o sexo e o peso ao nascer, mas a classe socioeconômica não esteve associada. Um estudo brasileiro com crianças de 1 ano de idade encontrou associação entre os despertares noturnos e o sexo do bebê e o fato de a mãe trabalhar fora de casa na gravidez, porém a idade materna, a escolaridade, o nível socioeconômico, a prematuridade, o baixo peso ao nascer e as intercorrências neonatais não estavam associados ao desfecho. Autores mostraram que fatores sociodemográficos e características da criança não possuem relação com as alterações do sono dos 6 aos 12 meses $^{9,20}$.

As mães deprimidas desta amostra apresentam maior probabilidade de possuir um filho com problemas de sono. Outros estudos corroboram esses achados ${ }^{6,9,14,15,21}$, mas não foi encontrado nenhum estudo brasileiro que avaliasse a associação entre o quadro depressivo materno e os comportamentos de sono infantil. A hipótese de que as mães deprimidas encontram mais dificuldades para regular o afeto, compreender e responder adequadamente as necessidades dos seus bebês e são mais inconsistentes e ineficazes no manejo com a criança deve ser considerada, uma vez que esses aspectos teriam um papel fundamental na regularização dos padrões do sono ${ }^{6}$.

Entretanto, a depressão paterna aos 12 meses nesta amostra não interfere na consolidação dos padrões de sono do bebê e, à semelhança a outras pesquisas, não encontrou associação̊ 
Nesta amostra, os bebês que sofreram algum tipo de acidente são mais propensos a possuírem alterações no sono. Não foram localizados outros estudos que tenham avaliado a relação entre traumas físicos e sono de bebês.

Algumas limitações devem ser apontadas: a) os comportamentos de sono das crianças foram investigados por meio do relato dos pais e a depressão pode alterar a percepção dos comportamentos; porém, na tentativa de minimizar esse viés, o questionário foi respondido pelo pai e pela mãe conjuntamente; b) não existe no Brasil um instrumento já validado para avaliar essas alterações de sono, por esse motivo as perguntas foram baseadas em achados de outras investigações; c) a interpretação dos dados é limitada pelo desenho do estudo transversal, não podendo ser feita uma relação causa-efeito entre as alterações do sono em bebês e as demais variáveis; d) o possível viés de causalidade reversa: um modelo causal foi testado em outra investigação e os pesquisadores concluíram que é improvável que os despertares noturnos levem à depressão materna; entretanto, os sintomas depressivos maternos podem contribuir para a ocorrência dos despertares noturnos ${ }^{6}$.

Essas limitações apontadas devem ser consideradas, porém não invalidam a indicação que é oferecida pelos resultados. Os profissionais de saúde devem investigar rotineiramente os comportamentos de sono de crianças pequenas e sua associação com a sintomatologia depressiva dessas mães, proporcionando uma identificação precoce de problemas e, consequentemente, a possibilidade de medidas curativas e preventivas para um adequado desenvolvimento infantil.

\section{CONCLUSÕES}

Para esta amostra, 35,7\% dos bebês apresentaram alteração no padrão de sono e pode-se perceber que a alteração no sono dos bebês manteve-se associada com a sintomatologia depressiva da mãe. Sendo assim, sugere-se que os profissionais de saúde devem investigar rotineiramente os comportamentos de sono dos bebês e dar atenção à saúde mental das mães, a fim de identificar os problemas precocemente e oferecer suporte no manejo do sono dos bebês.

\section{REFERÊNCIAS}

1. Lent R. Cem bilhões de neurônios: conceitos fundamentais de neurociência. São Paulo: Editora Atheneu; 2005.

2. Mindell J, Boaz A, Joffe M, Curtis S, Birley M. Evidence based public health policy and practice: enhancing the evidence base for health impact assessment. J Epidemiol Community Health. 2004;58:546-55.

3. Moore T, Ucko LE. Nigth waking in early infancy. Arch Dis Child. 1957;32(164):333-42.

4. Mazet P, Braconnier A. 0 sono das crianças e seus problemas. Rio de Janeiro: Editora Bertrand Brasil; 1993.

5. Bee H. 0 ciclo vital. Porto Alegre: Artes Médicas; 1997.

6. Warren SL, Howe G, Simmens SJ, Dahl RE. Maternal depressive symptoms and child sleep: models of mutual influence over time. Dev Psychopathol. 2006;18(1):1-16.

7. Müller MR, Guimarães SS. Sleep disorders impact on daily functioning and life quality. Estudos de Psicologia. 2007;24(4):519-28

8. Martin J, Hiscock H, Hardy P, Davey B, Wake M. Adverse associations of infant and child sleep problems and parent health: an Australian population study. Pediatrics. 2007;119(5):947-55.

9. Hiscock H, Wake M. Infant sleep problems and postnatal depression: a community-based study. Pediatrics. 2001;107(6):1317-22.

10. Field T. Infants of depressed mothers. Infant Behav Dev. 1995;8:1-13.

11. Pinheiro RT, Magalhães PV, Horta BL, Pinheiro KA, Da Silva RA, Pinto RH. Is paternal postpartum depression associated with maternal postpartum depression? Population-based study in Brazil. Acta Psychiatr Scand. 2006;113(3):230-2.

12. Dennis CL, Ross L. Relationships among infant sleep patterns maternal fatigue, and development of depressive symptomatology. Birth. 2005;32(3):187-93.

13. Paulson JF, Dauber S, Leiferman JA. Individual and combined effects of postpartum depression in mothers and fathers on parenting behavior. Pediatrics. 2006;118(2):659-68.

14. Bayer JK, Hiscock H, Hampton A, Wake M. Sleep problems in young infants and maternal mental and physical health. J Paediatr Child Health. 2007;43(1-2):66-73.

15. Meltzer LJ, Mindell JA. Relationship between child sleep disturbances and maternal sleep, mood, and parenting stress: a pilot study. J Fam Psychol. 2007;21(1):67-73.

16. Dearing E, McCartney K, Marshall NL, Warner RM. Parental reports of children's sleep and wakefulness: longitudinal associations with cognitive and language outcomes. Infant Behav Dev. 2001;24:151-70.

17. Gregory AM, O'Connor TG. Sleep problems in childhood: a longitudinal study of developmental change and association with behavioral problems. J Am Acad Child Adolesc Psychiatry. 2002:41(8):964-71.

18. Santos IS, Matijasevich A, Tavares BF, Barros AJ, Botelho IP, Lapolli C, et al. Validation of the Edinburgh Postnatal Depression Scale (EPDS) in a sample of mothers from the 2004 Pelotas Birth Cohort Study. Cad Saude Publica. 2007;23(11):2577-88.

19. Associação Brasileira de Empresas em Pesquisa (ABEP). Critério de classificação econômica no Brasil. Disponível em: http://www.abep.org/codigosguias/ABEP_CCEB.pdf. Acessado em: dez. 2008

20. Santos IS, Mota DM, Matijasevich A. Epidemiology of co-sleeping and nighttime waking at 12 months in a birth cohort. J Pediatr (Rio J). 2008;84(2):114-22.

21. Wake M, Allen EM, Poulakis Z, Hiscock H, Gallagher S, Oberklaid F. Prevalence, stability, and outcomes of cry-fuss and sleep problems in the first 2 years of life: prospective community-based study. Pediatrics. 2006;117(3):836-42. 